\title{
Use of the Brief Observation of Symptoms of Autism (BOSA) as a new clinical approach to assessing patients with suspected spectrum disorder during the COVID-19 pandemic
}

\author{
Agnieszka Rynkiewicz' ${ }^{1}$ Roma Vasa² ${ }^{2}$ Izabela Łucka³ ${ }^{3}$ Artur Mazur \\ 'Department of Psychiatry, Institute of Medical Sciences, Medical College of Rzeszów University, Poland \\ ${ }^{2}$ Kennedy Krieger Institute, Department of Psychiatry and Behavioral Sciences, Johns Hopkins University School of Medicine, \\ USA \\ ${ }^{3}$ Department of Developmental Psychiatry, Psychotic and Geriatric Disorders, Medical University of Gdańsk, Poland \\ ${ }^{4}$ Department of Paediatrics, Institute of Medical Sciences, Medical College of Rzeszów University, Poland
}

\section{ABSTRACT}

COVID-19 has imposed major changes to the delivery of healthcare worldwide. In many settings, healthcare is now predominantly transitioned to telehealth, creating challenges and unique benefits. In 2020, the Centers for Disease Control and Prevention has reported that 1 in 54 children in the U.S. are diagnosed with autism spectrum disorder (ASD). The Brief Observation of Symptoms of Autism (BOSA) is a new clinical approach that has been developed to ensure that patients suspected of ASD can receive valid diagnostic assessments during the pandemic. BOSA is offered when a diagnostician is unable to administer the Autism Diagnostic Observation Schedule - Second Edition (ADOS-2) either because it is not appropriate to administer the ADOS-2 via telehealth or, in person, face coverings confound ASD assessment. Only trained ADOS-2 diagnosticians can administer BOSA, because BOSA relies on ADOS- 2 codes. The article describes BOSA to prepare the field for future research during the COVID-19 crisis.

\section{KEY WORDS:}

COVID-19 pandemic, BOSA, ADOS-2, autism spectrum disorder.

\section{INTRODUCTION}

Autism spectrum disorder (ASD) is no longer a rare neurodevelopmental condition [1]. Recent estimates from the Centers for Disease Control and Prevention indicate that 1 in 54 children in the U.S. are diagnosed with ASD [1]. ASD is a lifelong disability, and symptoms are usually apparent from early childhood. The COVID-19 pandemic, caused by the novel coronavirus SARS-CoV-2, has affected countries globally [2]. The pandemic has unfortunately left many patients suspected of having ASD and their families with very limited access to both diagnostic services and developmental therapies. The COVID-19 pandemic has also imposed changes to the health system worldwide. Healthcare has now transitioned to telehealth models, creating both challenges and unique benefits for families awaiting an ASD evaluation for their child. To address the critical need for early diagnosis and treatment of ASD, the Brief Observation of Symptoms of Autism (BOSA) has been developed to ensure that patients suspected of ASD receive timely and valid assessments during the COVID-19 pandemic [3]. The BOSA was de-

\section{ADDRESS FOR CORRESPONDENCE:}

dr n. med. Agnieszka Rynkiewicz, Zakład Psychiatrii, Instytut Nauk Medycznych, Kolegium Nauk

Medycznych, Uniwersytet Rzeszowski, Rzeszów, Poland, ORCID: 0000-0002-9175-4937,

e-mail: arynkiewicz@ur.edu.pl 
veloped by Dr. Catherine Lord [3], who is the co-author of Autism Diagnostic Observation Schedule (ADOS-2) $[4,5]$ and her team [3]. The ADOS-2 along with the Autism Diagnostic Interview - Revised (ADI-R) [6] are considered gold standard instruments to supplement the diagnosis of ASD [7]. BOSA is based on activities and a subset of codes that have been selected and adapted from the ADOS-2 and Brief Observation of Social Communication Change (BOSCC), another well-validated assessment measure of social communication skills $[8,9]$. The BOSA is a tool that has been developed to assist diagnosticians in their ASD diagnostic decision-making during the pandemic. It is not intended to replace the ADOS-2 but rather to supplement the clinical evaluation. BOSA can be offered when a diagnostician is not able to administer a valid ADOS-2 during the pandemic because it is either not feasible via telehealth or when the parties involved are wearing face coverings that preclude precise symptom measurement [3]. BOSA is in the first stages of psychometric evaluation, and research is underway looking at the predictive utility of BOSA using clinical (ASD) and community (neurotypical) samples. All queries for access and use of BOSA and its available language translations for research purposes need to be directed to Making Better Measures [3]. This new measure which is BOSA and also ADOS- 2 and BOSCC are copyrighted by WPS [10] and C. Lord [3]. The article describes BOSA to make the field for future research with this new assessment during the COVID-19 crisis.

\section{WHO CAN ADMINISTER THE BRIEF OBSERVATION OF SYMPTOMS OF AUTISM?}

Only trained and experienced ADOS-2 diagnosticians can administer the BOSA because the BOSA relies on ADOS- 2 codes. Diagnosticians are not required to be research reliable in ADOS-2 administration; however, they should have sufficient experience with administering the ADOS-2 and establishing a diagnosis of ASD. Diagnosticians are required to attend BOSA training before they start using this measure [3]. Modifications of the BOSA materials are prohibited. The ADOS-2 protocols are used during the BOSA observations. Only ADOS-2 diagnosticians trained in the BOSA can score the measure.

\section{WHAT ARE THE VERSIONS OF THE BRIEF OBSERVATION OF SYMPTOMS OF AUTISM?}

The BOSA has been developed based on the standardised activities adapted from two well-validated assessments: the BOSCC and the ADOS-2. The activities have been so adapted that a parent or therapist can engage the child via telehealth. These social interaction activities provide opportunities to create a context in which a diagnostician can observe symptoms of ASD. The BOSA takes approximately 15 minutes to administer. There are four BOSA versions. Diagnosticians select an appropriate version based on the individual's age and language level: 1. BOSA-MV (minimally verbal): appropriate for any age, for minimally verbal participant (i.e. a patient who is nonverbal or uses only single words or phrases).

2. BOSA-PSYF (phrase speech and young fluent): appropriate for any age, for participants who use flexible phrase speech or verbally fluent children under the age of 6-8 years.

3. BOSA-F1 (fluent speech F1): appropriate for verbally fluent children aged 6-8 years up to age 10 years.

4. BOSA-F2 (fluent speech F2): appropriate for verbally fluent children ages 11 years and older and also verbally fluent adults.

\section{HOW IS THE BRIEF OBSERVATION OF SYMPTOMS OF AUTISM ADMINISTERED?}

A diagnostician can observe the BOSA live or via a recorded video and then complete selected subset of ADOS-2 codes based on this observation. Observations can be done in several ways: through a one-way mirror in the observation room, via telehealth (e.g. Zoom platform, or a video-transmission from another clinic), a diagnostician can be in the same room as a participant while maintaining the required social distancing, observation can be in the participant's home, or a diagnostician can watch and score the observation based on video material that was recorded earlier and then submit scores for evaluation.

The first step a diagnostician decides on is the BOSA version to be used. The next steps are to prepare the environment for the session and arrange the room, people, and materials. The diagnostician then explains their role in the assessment process and reviews the instruction sheet together with the parent/caregiver or therapist who will be presenting the activities. The diagnostician (i.e. caregiver/therapist) should look through the materials/toys and get familiar with them before beginning the session. The BOSA uses the ADOS-2 kit and several supplemental materials. The session begins with a caregiver/therapist taking out a few toys that are the most enjoyable and motivating for the child, to help them engage the child in play. The remaining toys are put away and out of reach. The parent/caregiver can also bring the child's own toys to help them engage in a session. The caregiver/therapist plays some games and talks with the participant during the session with the adults with fluent speech. In addition to the games there are question cards for both the examiner and participant, which open the conversation to include, for example, emotions or social difficulties.

After the BOSA session has been completed, the diagnostician uses the ADOS-2 protocols for scoring. The binary ADOS-2 scoring is used because the BOSA has been adapted from the standardised activities of the ADOS-2 and BOSCC. Additionally, the diagnostic and statistical manual of mental disorders, fifth edition (DSM-5) [11] or 
ICD-10 [12] checklists are used, which have been specifically created for this assessment, and several inexpensive supplemental materials that are not part of the ADOS-2 kit and can be purchased [3]. For sanitation purposes, certain ADOS-2 materials should be replaced after each session (e.g. plastic baby doll) while the other materials can be sanitised, which is a practical yet safe approach (e.g. Jenga game or waterproof cards to play Slap Jack). After the BOSA session has been scored, even experienced diagnosticians may need additional information to confirm or rule out autism. This information could be obtained from ongoing longitudinal assessment and/ or administration of other ASD assessment tools such as the ADI-R. It is stressed here that when a diagnostician is uncertain about the diagnosis despite having multiple sources of information, a diagnosis of ASD should be deferred until a follow-up evaluation can be conducted in person, at which the ADOS- 2 and clinical evaluation can be conducted with greater accuracy.

\section{LIMITATIONS OF THE BRIEF OBSERVATION OF SYMPTOMS OF AUTISM}

BOSA has been developed in response to an urgent need to avoid delays in ASD diagnosis during the COVID-19 pandemic; however, there are several limitations that should be noted. BOSA is in the first stages of psychometric evaluation, and research is underway looking at the predictive utility of BOSA using ASD and neurotypical samples. There is still unknown sensitivity and specificity. This new measure has not been validated yet, and there are no empirically derived cut-offs currently available. Therefore, diagnosticians should integrate the final score within the context of a clinical evaluation that uses the DSM-5 diagnostic framework for ASD. Key domains of the clinical evaluation must include a thorough developmental, medical, and psychiatric history. Ideally, the BOSA should be supplemented with the ADI-R [6], a diagnostician administered measure that can be done via telehealth, e.g. Zoom platform with a parent/caregiver, which is beneficial during the pandemic. The reliance on parent behaviour is the other limitation, and this area needs thorough scientific research. However, a diagnostician experienced in autism can find a lot of valuable information for his/her clinical and therapeutic judgment based on the observation of this interaction between a parent and child even if it cannot be scored in the context of BOSA. Kanne and Bishop [13] encourage the development of innovative diagnostic models that include the appropriate roles for non-specialty providers but emphasise the accuracy and quality of patient care. The BOSA as the supplemental solution in the current COVID-19 pandemic can be beneficial but only if the interpretation of the scores is done by well-trained ADOS-2 and ADI-R expert providers; only then does this model not come at the expense of quality.
Researchers and clinicians worldwide can collaborate on providing scientific data for BOSA and working diligently to provide easy access to BOSA as quickly as possible to ensure that patients who are suspected of having ASD and their families are not left without appropriate assessments during these uncertain times.

\section{DISCLOSURE}

The authors declare no conflict of interest.

\section{REFERENCES}

1. Centers for Disease Control and Prevention (CDC). 2020 community report on autism. https://www.cdc.gov/ncbddd/autism/ addm-community-report/documents/addm-community-report2020-h.pdf (accessed: 17.08.2020).

2. World Health Organization. Coronavirus disease (COVID-19) pandemic. https://www.who.int/health-topics/coronavirus\#tab=tab_1 (accessed: 17.08.2020).

3. Lord C, Holbrook A, Dow D, et al. Brief Observation of Symptoms of Autism (BOSA). Version: 7-28-2020. https://www.semel.ucla. edu/autism/bosa-training (accessed: 29.07.2020).

4. Lord C, Rutter M, DiLavore PC, et al. Autism diagnostic observation schedule (ADOS). Western Psychological Services, Los Angeles, CA 1999.

5. Lord C, Rutter M, DiLavorne PC, et al. Autism diagnostic observation schedule, second edition (ADOS-2) manual (Part I): modules 1-4. Torrance, Western Psychological Services, Los Angeles, CA 2012.

6. Rutter M, Le Couteur A, Lord C. Autism diagnostic interview revised (ADI-R). Western Psychological Services, Los Angeles, CA 2003.

7. Rynkiewicz A, King BH, Kalisz K, et al. Competent users and standards of use for autism diagnostic interview - revised (ADI-R) and autism diagnostic observation schedule, second edition (ADOS-2). Psychiatr Psychol Klin 2018; 18: 399-404.

8. Grzadzinski R, Carr T, Colombi C, et al. Measuring changes in social communication behaviors: preliminary development of the Brief Observation Of Social Communication Change (BOSCC). J Autism Develop Dis 2016; 46: 2464-2479.

9. Grzadzinski R, Lord C. Commentary: insights into the development of the Brief Observation Of Social Communication Change (BOSCC). J Mental Health Clin Psychol 2018; 2: 15-18.

10. Western Psychological Services, WPS. https://www.wpspublish. com/ (accessed: 29.07.2020).

11. American Psychiatric Association. Diagnostic and statistical manual of mental disorders: DSM-5. Arlington, VA: American Psychiatric Association 2013.

12. The ICD-10 classification of mental and behavioural disorders: clinical descriptions and diagnostic guidelines. World Health Organization, Geneva 1992.

13. Kanne S, Bishop S. Editorial perspective: the autism wait-list crisis and remembering what families need. J Child Psychol Psych 2020. https://doi.org/10.1111/jcpp.13254. 